\title{
Clinical features of intratarsal keratinous cysts
}

\begin{abstract}
Purpose Intratarsal keratinous cysts (IKCs) have been frequently misdiagnosed as chalazia or epidermal cysts. We reviewed a series of cases of IKCs to identify clinical features that distinguish IKCs from other eyelid diseases.

Methods We retrospectively reviewed the medical records of 17 suspected IKC patients between January 2004 and September 2014. Results Seventeen patients who were clinically suspected to have IKC were enrolled. All patients presented with noninflamed eyelid nodules fixed to the tarsus. Among them, 12 biopsy specimens were available and 11 patients $(91.7 \%)$ were diagnosed with IKC, with a pathological finding of stratified squamous cell lining with keratin material. The mean patient age was 55.1 years (31-71). Six patients had a surgical history of incision or incomplete excision of the lesion, followed by recurrence. On eyelid eversion, five patients showed a white-yellow nodule, and three patients had a bluish cystic lesion. The diameter of the nodules ranged from 4 to $10 \mathrm{~mm}$. The intracystic material was a milky white fluid. Ten patients underwent a complete surgical excision including partial tarsectomy and there was no recurrence. Conclusions IKC can be distinguished from other intratarsal lesions by a characteristic tarsal nodule fixed to the tarsus. To prevent recurrence, complete excision with partial tarsectomy is needed.

Eye (2016) 30, 59-63; doi:10.1038/eye.2015.184; published online 2 October 2015
\end{abstract}

\section{Introduction}

Most common primary intratarsal lesions are chalazia and sebaceous gland carcinomas. ${ }^{1}$ An intratarsal keratinous cyst (IKC), recently described by Jakobiec et al, ${ }^{2}$ is an intratarsal mass lesion that originates from the meibomian glands or their ducts. Importantly, IKC has been frequently misdiagnosed as chalazion or other

JA Kim ${ }^{1}$, N Kim², H-K Choung ${ }^{3}$, MJ Lee ${ }^{4}$, C Lee ${ }^{5}$ and SI Khwarg ${ }^{1}$

benign eyelid cystic tumors because it is an intratarsal hard nodular mass. It is important to differentiate IKC from other eyelid intratarsal lesions because its therapeutic approach and clinical outcomes are quite different; IKC has to be completely excised with partial tarsectomy. In contrast, a chalazion is easily treated with a simple incision and curettage (I\&C). Sebaceous gland carcinomas often metastasize and contribute to mortality despite radical surgical excision. Some reports ${ }^{3,4}$ have highlighted the histopathological features of IKC. However, there is no published report of the correlation between clinically suspected IKC and confirmed diagnosis of IKC by pathological examination. In this study, we report on 11 cases of histologically confirmed IKC and their clinical features.

\section{Materials and methods}

Patients who underwent surgical treatment for eyelid masses at four different medical centers from January 2004 to September 2014 were recruited, and their medical records and photographs were retrospectively reviewed. The records of patients who were assumed to have IKC were collected and their pathologic slides were re-examined by an experienced pathologist (CL). The data included sex, age at the time of excision, location, color and size of the lesions, related symptoms, duration of symptoms, findings on external examination and eyelid eversion, previous diagnosis, treatment, intraoperative findings, previous history of treatment, follow-up duration, and recurrence.

This study complied with the Declaration of Helsinki guidelines and was approved by the Institutional Review Board of Seoul Metropolitan Government Seoul National University Boramae Medical Center, Seoul, Korea.

\section{Results}

Seventeen patients who were clinically suspected to have IKC were enrolled. All

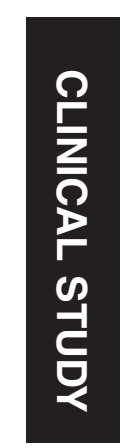

${ }^{1}$ Department of

Ophthalmology, Seoul

National University College of Medicine, Seoul, Korea

${ }^{2}$ Department of Ophthalmology, Seoul National University Bundang Hospital, Seongnam, Korea

${ }^{3}$ Department of Ophthalmology, Seoul Metropolitan Government-Seoul National University Boramae Medical Center, Seoul, Korea

${ }^{4}$ Department of Ophthalmology, Hallym University Sacred Heart Hospital, Anyang, Korea

${ }^{5}$ Department of Pathology, Seoul National University College of Medicine, Seoul, Korea

Correspondence: H-K Choung, Department of Ophthalmology, Seoul Metropolitan GovernmentSeoul National University Boramae Medical Center, \#20 Boramae-ro 5-gil, Dongjak-gu, Seoul 156-707, Korea

Tel: +82 2870 2412;

Fax: +82 28310714 . E-mail: hokyung214@ gmail.com

Received: 2 March 2015 Accepted in revised form: 25 July 2015

Published online:

2 October 2015

This paper has not been presented in any academic conference. 
patients presented with non-inflamed firm nodules fixed to the tarsus. Among them, the biopsy specimens of 12 patients were available and 11 patients $(91.7 \%)$ were given a confirmed diagnosis of IKC by pathological examination (Table 1). The mean patient age was 55.1 years (range 31-71 years), and five patients were men and six were women. The overlying skin was freely movable without focal adhesion. All mass lesions were located at the upper eyelid. On eyelid eversion, well-demarcated upraised intratarsal lesions were found through the conjunctiva, and the surrounding conjunctiva was not inflamed. The nodules had a diameter of $\sim 4-10 \mathrm{~mm}$, with a mean diameter of $6.6 \mathrm{~mm}$ (Table 1). Five patients showed a white to yellow nodular bulge and three patients showed a bluish cystic lesion (Figure 1). All IKCs were solitary except one in which multiple lesions distorted the eyelid margin. One clinically misdiagnosed patient was diagnosed as having an epidermal cyst. Six patients had a history of incision or incomplete excision followed by recurrence.

Previously, before recognition of IKC as an entity, patients were clinically diagnosed as having another disease; two patients as having a chalazion, three patients as having an epidermal cyst, and four patients as having a tarsal mass. Pathological examination, which was performed on 12 patients prior to the discovery of IKC, indicated that 3 patients had an epidermal inclusion cyst, 3 patients had a steatocystoma, 2 patients had a keratinous cyst, 1 patient had a cyst of Moll's gland, 1 patient had a pilar cyst, and 1 patient had a benign cyst with fibrovascular tissue. After review by an experienced pathologist, all cases except one were re-diagnosed as IKC (Figure 2).

Ten patients underwent surgical excision including partial tarsectomy: four patients through a transcutaneous approach, four patients through a transconjunctival approach, and two patients through an unrecorded approach. We did not suture the conjunctiva after using the transconjunctival approach and there were no corneal complications. Six cases were ruptured during dissection and a milk-like fluid mixed with solid elements having the consistency of tofu residue, which is different from the ordinary content of chalazia, was extruded. None of the patients experienced recurrence during the follow-up, which had a mean duration of 5 weeks (range, 1-24 weeks). Among the 12 patients who were clinically suspected as having IKC, only one patient was ultimately pathologically diagnosed with an epidermal cyst. In this patient, an intratarsal gray-black mass was also observed on eyelid eversion. However, on pathological examination, the cyst was lined by focal acanthotic squamous epithelium and had a granular layer in the cyst wall. Thus, these findings were indicative of an epidermal cyst, rather than IKC.

\section{Discussion}

IKCs are a recently described entity that arises from the Meibomian glands and are thought to be the third major cause of intratarsal swelling after chalazia and sebaceous cell carcinomas. ${ }^{2}$ The clinical characteristics of IKCs are similar to those of chalazia and epidermal inclusion cysts, but there are differentiating features that enable physicians to distinguish them. For example, lesions such as steatocystomas and pilomatrixomas are white to yellow, as are IKCs. In contrast, although chalazia are fixed to the tarsus similarly to IKC, they are frequently accompanied by surrounding inflammation and the margin of the lesion is less clear than that of an IKC. These features are helpful for differentiation of chalazia from IKC. ${ }^{5}$ An epidermal inclusion cyst is a non-inflammatory, palpable nodule that is not fixed to the tarsus but is freely movable, which is another differentiating characteristic with regard to IKC. ${ }^{6}$ The cyst content of a steatocystoma is a yellowish oily sebum containing hair shafts, and steatocystomas are not attached to the tarsus. ${ }^{7}$ Only two cases of pilomatrixoma observed at the tarsal conjunctiva have been reported. ${ }^{8,9}$ Usually, pilomatrixoma grows toward the skin and is freely movable from the tarsus because it is thought to originate from hair follicle cells. Furthermore, patients with pilomatrixoma are usually young, and hyperemia is observed surrounding the lesion, in contrast to IKC. IKCs occasionally have a bluegray color because of the Tyndall effect, and a blue IKC is occasionally misdiagnosed as melanoma. ${ }^{2}$

As awareness of IKC increases, histopathological differences between this entity and other diseases are being revealed. Even though IKC and steatocystoma share similar histological findings, such as trichilemmal keratinization, steatocystomas contain sebaceous glands in the cyst wall or adjoining wall. However, for most cases, a careful clinical examination can provide sufficient evidence for a differential diagnosis. With the aforementioned clinical characteristics in mind, we reviewed medical photographs and records to identify cases that were previously diagnosed as chalazia or epidermal cysts and were now clearly re-diagnosed as IKC. As a result, among 12 patients who were clinically suspected as having IKC, only one patient was ultimately pathologically diagnosed with an epidermal cyst.

Previously, IKC was a commonly misdiagnosed lesion. However, with further investigation, we found that most cases were correctly diagnosed as IKC.

Regarding pathogenesis, Lucarelli et al ${ }^{10}$ suggested that eyelid surgery or trauma may cause a blockage of the tarsal sebaceous ducts and squamous metaplasia, leading to the development of IKCs. In our study, there were eight cases of tarsal nodule excision or incision and one case of a double eyelid operation, which occurred $\sim 23$ 


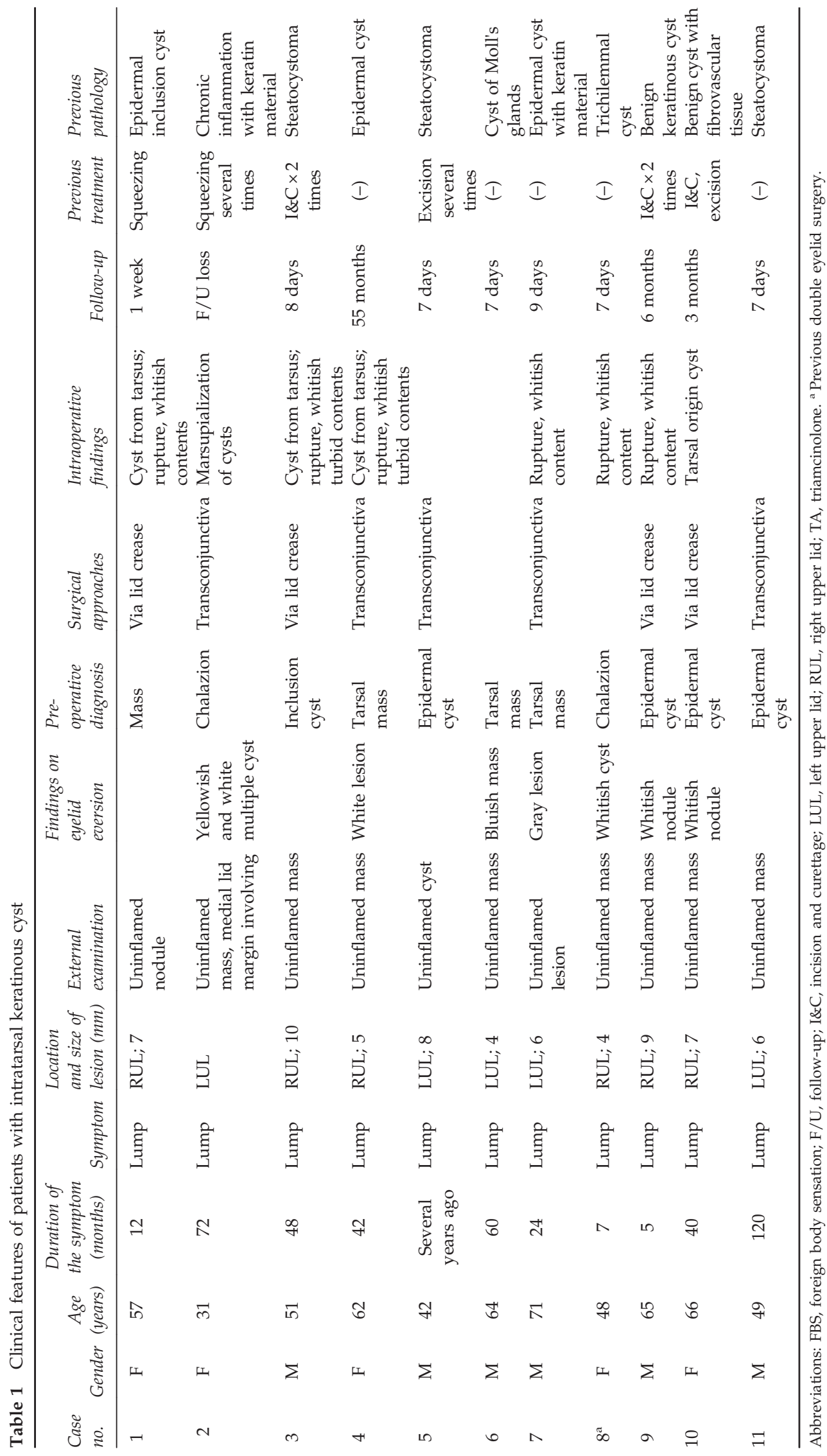



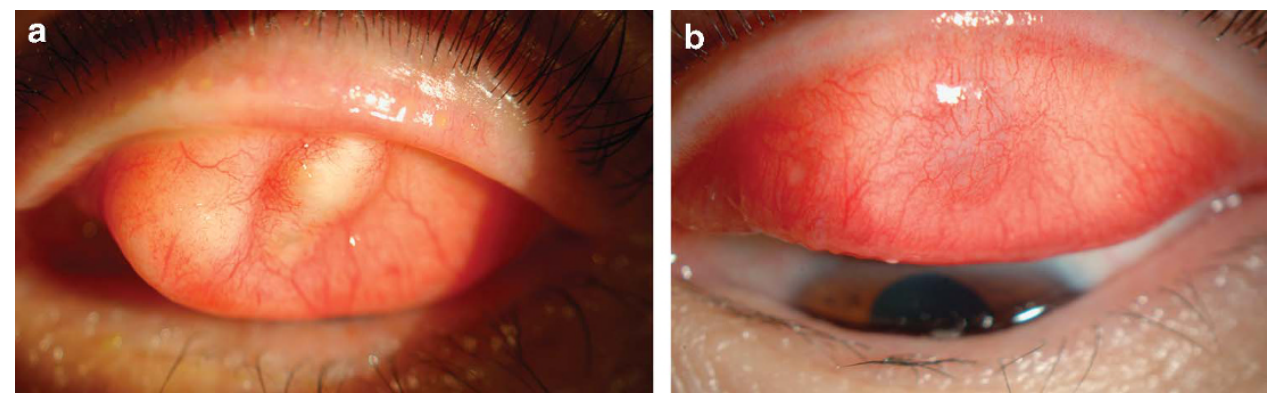

Figure 1 Photographs of intratarsal keratinous cysts. (a) Whitish, 6-mm, elevated nodule without signs of inflammation. (b) Blue, 8-mm, palpable intratarsal nodule.
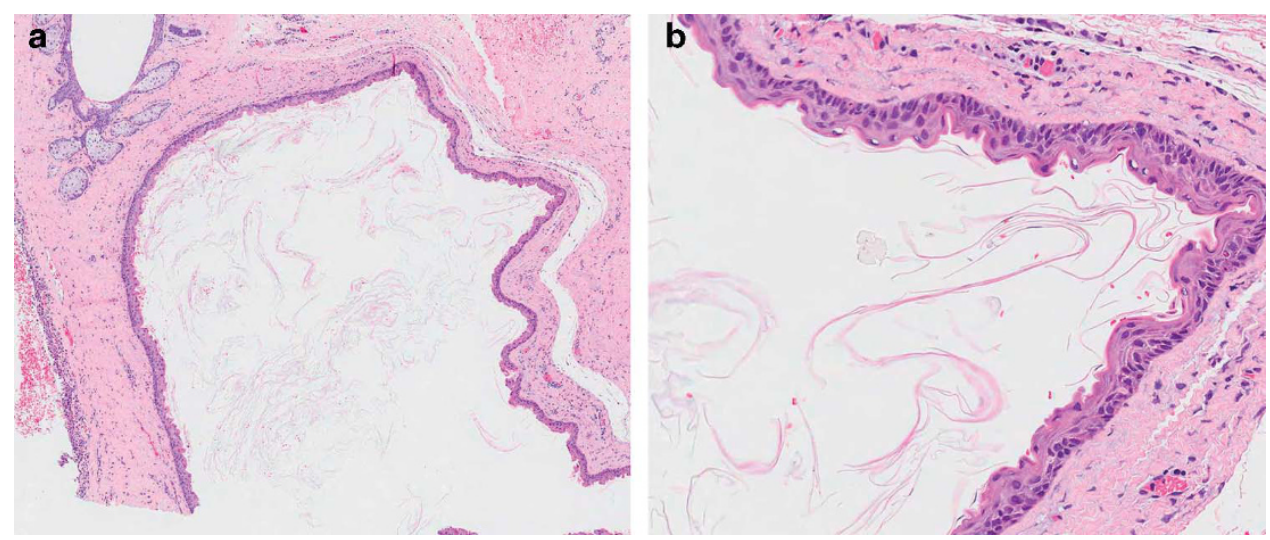

Figure 2 Histological findings of an intratarsal keratinous cyst. (a) Benign keratinous cyst adjacent to the Meibomian gland (Hematoxylin-eosin stain, original magnification: $\times 40$ ). (b) The cyst lined by undulating squamous epithelium without the granular layer (Hematoxylin-eosin stain, original magnification $\times 200$ ).

years ago. However, Jakobiec et $a l^{2}$ reported that IKC consists of a firm fibrous cyst and intracystic content, and that the fibrous wall of the cyst is composed of tightly woven bundles of collagen. They also reported that the ducts and ductules of the Meibomian glands were composed of non-keratinizing squamous epithelium, and there was no histological evidence of squamous metaplasia. Therefore, we did not exclude the patient who had a previous double eyelid operation from this study.

In our study, there was one case with multiple lesions. In 2012, Kim et al ${ }^{11}$ reported multiple IKCs of the eyelid for the first time. Subsequently, to our knowledge, there has been no report of multiple IKCs, and thus, we assume that multiple IKCs are uncommon. Furthermore, we could not find a cause for the multiple cysts in our case, which is consistent with the case of Kim et al Further observation would be needed to understand the mechanism of multiple lesions.

Therapeutically, it is important to distinguish IKC from other similar lesions because IKC requires complete excision. There are many cases of multiple recurrences that were previously diagnosed as chalazia and subsequently operated on with an incomplete excision or I\&C. The multiple recurrences of chalazia suggest that the diagnosis was incorrect. To excise an IKC, a partial tarsectomy is mandatory because of its firm adhesion to the tarsus, and this procedure that will remarkably reduce recurrence. ${ }^{2}$ Surgically, there are following two approaches: transcutaneous and transconjunctival. Patel et $a l^{3}$ reported that an anterior lid crease approach with full excision of the intratarsal cyst and partial excision of the underlying anterior tarsus is effective for preventing local recurrence and avoiding more complex lid reconstruction. In addition, Zhang et al ${ }^{4}$ suggested that a transconjunctival incision avoids an eyelid incision and disfiguration. They suggested that because the IKC is fixed to the tarsus and is independent of the skin, the conjunctival approach with partial tarsectomy is a better way to excise the entire lesion and simultaneously avoid cosmetic problems. Comparisons of transconjunctival vs transcutaneous approaches for lower eyelid blepharoplasty or orbit wall fracture repair have been studied previously. However, no report has identified a better approach for tarsal lesions. In our study, there were 
four cases with a transcutaneous approach and four cases with a transconjunctival approach, and no patients reported dissatisfaction with the cosmetic outcomes. There was also no case of severe eyelid contour distortion or scar formation.

In this study, we identified 11 cases of IKC and their clinical features. It is important to distinguish IKC from other similar lesions because IKC requires complete excision. The results indicate that multiple recurrences after I\&C or incision and drainage of a noninflammatory, painless intratarsal mass suggest the presence of IKC.

\section{Summary}

What was known before

- IKC is thought to be the third major intratarsal mass.

- IKC shares similar features with other lesions, and it is important to distinguish IKC from these other lesions because IKC requires a complete excision.

\section{What this study adds}

- Typically, cases with clinical suspicion of IKC are actually IKC (11/12 confirmed histologically).

- Clinical suspicion of IKC therefore typically reflects the actual disease state and should be considered as such.

\section{Conflict of interest}

The authors declare no conflict of interest.

\section{References}

1 Spencer WH. Ophthalmic Pathology: an Atlas and Textbook, 4th edn. WB Saunders: Philadelphia, PA, USA, 1996; 2378-2380.

2 Jakobiec FA, Mehta M, Iwamoto M, Hatton MP, Thakker M, Fay A. Intratarsal keratinous cysts of the Meibomian gland: distinctive clinicopathologic and immunohistochemical features in 6 cases. Am J Ophthalmol 2010; 149(1): 82-94.

3 Patel VS, Meyer DR, Carlson JA. Intratarsal keratinous cysts of the meibomian gland (a sebaceous duct cyst): report of 2 cases. Am J Dermatopathol 2011; 33(6): 624-627.

4 Zhang ZD, Li X, Li M, Zhao J, Zhou KJ, Qu J. Clinicopathological features and surgical treatment of intratarsal keratinous cysts. Am J Dermatopathol 2013; 35(1): 78-82.

5 Dhaliwal U, Arora VK, Singh N, Bhatia A. Cytopathology of chalazia. Diagn Cytopathol 2004; 31(2): 118-122.

6 Dutton JJ, Fowler AM, Proia AD. Dermoid cyst of conjunctival origin. Ophthal Plast Reconstr Surg 2006; 22(2): 137-139.

7 Procianoy F, Golbert MB, Golbspan L, Duro KM, Bocaccio FJ. Steatocystoma simplex of the eyelid. Ophthal Plast Reconstr Surg 2009; 25(2): 147-148.

8 de Azevedo ML, Milani JA, de Souza EC, Nemer RS. Pilomatrixoma. An unusual case with secondary corneal ulcer. Arch Ophthalmol 1985; 103(4): 553-554.

9 Monshizadeh R, Cohen L, Rubin PA. Perforating follicular hybrid cyst of the tarsus. J Am Acad Dermatol 2003; 48(2 Suppl): S33-S34.

10 Lucarelli MJ, Ahn HB, Kulkarni AD, Kahana A. Intratarsal epidermal inclusion cyst. Ophthal Plast Reconstr Surg 2008; 24(5): 357-359.

11 Kim HJ, Wojno TH, Grossniklaus HE. Multiple intratarsal keratinous cysts of the eyelid. Ophthal Plast Reconstr Surg 2012; 28(5): e116. 